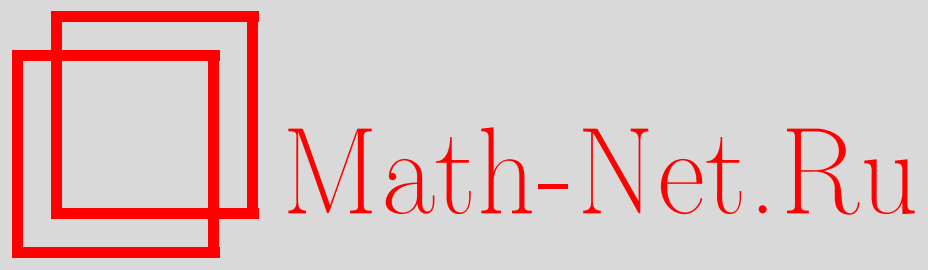

P. Zanzotto, Still on the semimartingales that are represented as stable integrals, Теория вероятн. и ее примен., 2003, том 48, выпуск 1, 198-204

DOI: https://doi.org/10.4213/tvp312

Использование Общероссийского математического портала Math-Net.Ru подразумевает, что вы прочитали и согласны с пользовательским соглашением

http://www . mathnet.ru/rus/agreement

Параметры загрузки:

IP: 34.229 .108 .108

26 апреля 2023 г., 10:16:34

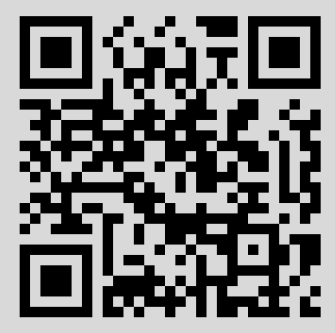




\title{
REFERENCES
}

1. Albeverio S., Rozanov Yu.A. On multi-period market extension with the uniformly equivalent martingale measure. - Acta. Appl. Math., 2001, v. 67, № 3, p. 253-275.

2. Dinculeanu $N$. Vector Measures. Oxford: Pergamon Press, 1967, $432 \mathrm{p}$.

3. Di Nunno G., Rozanov Yu. A. On monotone versions of Hahn-Banach extension theorem. - IAMI 99.11. Milano: CNR, 1999.

4. Fuchssteiner B., Lusky W. Convex Cones. Amsterdam: North-Holland, 1981, 429 p.

5. Kreps D. M. Arbitrage and equilibrium in economics with infinitely many commodities. - J. Math. Econom., 1981, v. 8, № 1, p. 15-35.

6. Schaefer H. H. Banach Lattices and Positive Operators. New York: Springer-Verlag, 1974, $376 \mathrm{p}$.

7. Yosida K. Functional Analysis. Berlin-Heidelberg: Springer-Verlag, 1980, 501 p.

Поступила в редакцию

9.VII. 2000

Исправленный вариант

14.V.2002

(C) $2003 \mathrm{r}$.

ZANZOTTO P. A.*

\section{STILL ON THE SEMIMARTINGALES THAT ARE REPRESENTED AS STABLE INTEGRALS}

\begin{abstract}
Приводится результат, дополняющий результаты предыдущей работы автора [7].

Ключевые слова и фразы: семимартингалы без непрерывной мартингальной составляющей, мера скачков, компенсатор, строго $\alpha$-устойчивые процессы Леви, устойчивые интегралы, формулы представления.
\end{abstract}

Introduction. In [7] we have characterized the one-dimensional semimartingales $X$ (with vanishing continuous martingale part) which can be represented as stochastic integrals (in the Itô sense) with respect to strictly $\alpha$-stable Lévy processes $Z^{(\alpha)}(0<\alpha<2)$, i.e., the semimartingales $X$ for which there exist a predictable process $H$ and a $Z^{(\alpha)}$ (possibly defined on some extension of the original probability space) that verify the following formulas:

$$
X_{t}=\int_{] 0, t]}\left|H_{s}\right| d Z_{s}^{(\alpha)}, \quad t \geqslant 0
$$

or

$$
X_{t}=\int_{] 0, t]} H_{s} d Z_{s}^{(\alpha)}, \quad t \geqslant 0 .
$$

Such characterization is essentially based on the following hypothesis which fixes the form of the compensator $\pi$ of the jump-measure $\mu$ of $X$ : there exist a predictable process $H$ and a constant $\alpha, 0<\alpha<2$, such that

$$
\pi(d s, d x)=\left|H_{s}\right|^{\alpha} d s \otimes \nu(d x)
$$

where $\nu$ is the Lévy measure of an $\alpha$-stable Lévy motion (this is a synonym of strictly $\alpha$-stable Lévy process).

* Facoltà di Economia dell'Università, Via F. Tomadini, 30/A, 33100 Udine, Italy; e-mail: zanzotto@dm.unipi.it 
In the case $0<\alpha<1$ (resp., $1<\alpha<2$ ) the class of semimartingales $X$ relevant to the formulas (1) and (2), is defined in an intrinsic way as that of process of pure jump type with locally finite variation paths (resp., that of purely discontinuous local martingales): denote these classes by $\mathscr{V}, \mathscr{L}$ respectively. Theorem 2 in [7] claims the following fact. Consider $X \in \mathscr{V}$ (resp., $X \in \mathscr{L}$ ) and assume that condition (3) holds for it with $\alpha$, $0<\alpha<1$ (resp., $1<\alpha<2$ ).

Then there is an $\alpha$-stable Lévy motion $Z^{(\alpha)}$ (possibly defined on some extension of the original probability space) with Lévy measure $\nu$, such that the representation formula (1) holds.

If $\nu$ in (3) is symmetric, besides (1) also the representation formula (2) holds in the same sense.

On the other hand, when (1) or (2) holds with $\alpha, 0<\alpha<1$ (resp., $1<\alpha<2$ ) then necessarily $X \in \mathscr{V}$ (resp., $X \in \mathscr{L}$ ).

And for all $\alpha, 0<\alpha<2$, condition (3) is necessary for the formula (1); it is necessary also for the formula (2) when the driving process $Z^{(\alpha)}$ has symmetric Lévy measure $\nu$. Thus in particular when $\alpha=1,(3)$ is necessary for both formulas (1), (2).

Coming now to the case $\alpha=1$, in [7] the analysis is restricted to the class $\mathscr{C}$, resp., $\mathscr{C}^{\prime}$, of semimartingales $X$ which admit the following decomposition

$$
X_{t}=\int_{00, t]} \int_{|x| \leqslant 1} x(\mu-\pi)(d s, d x)+\int_{[0, t]} \int_{|x|>1} x \mu(d s, d x)+b \int_{[0, t]}\left|H_{s}\right| d s,
$$

resp.,

$$
X_{t}=\int_{[0, t]} \int_{|x| \leqslant 1} x(\mu-\pi)(d s, d x)+\int_{j 0, t]} \int_{|x|>1} x \mu(d s, d x)+b \int_{] 0, t]} H_{s} d s,
$$

$b$ denoting a real number.

Then Theorem 1 in [7] claims the following property.

Consider $X \in \mathscr{C}$ (resp., $X \in \mathscr{C}^{\prime}$ ) and suppose that it verifies condition (3) with $\alpha=1$. Then the representation formula (1) (resp., (2)) holds with $\alpha=1, Z^{(1)}$ denoting a 1-stable Lévy motion with drift $b \cdot t$ and Lévy measure $\nu(d x)$ (defined in general on an extension of the original probability space).

Now one may wonder if there are semimartingales $X$ not in the class $\mathscr{C}$ (resp., $\mathscr{C}^{\prime}$ ) for which the representation formula (1) (resp., (2)) with $\alpha=1$ holds all the same. Here in Section 1 we show that this is not the case. In fact, besides condition (3) (with $\alpha=1$ ), also the decomposition form (4) (resp., (5)) is necessary for the semimartingales $X$ that are represented according to (1) (resp., (2)) with $\alpha=1, b$ denoting the drift coefficient of $Z^{(1)}$ : in other words for such semimartingales the decomposition (4) (resp., (5)) is intrinsic.

Further, concerning the proof of the results in [7], below in Section 2 we show a more elementary way of proceeding, using essentially only the concept of compensator of a random measure. Finally we want to point out that the representation properties in [7] constitute a tool for the results in [8].

1. Decomposition of 1-stable stochastic integrals. In the following we adopt the notation, definitions and assumptions of [7] to which the reader is referred.

In particular, everywhere below we denote by $(X, \mathbf{F})$ a real-valued $\mathbf{F}$-adapted process defined on $\mathbf{B}=(\Omega, \mathscr{F}, \mathbf{F}, \mathbf{P})$, by $\mu$ (resp., $\pi)$ the jump-measure of $X$ (resp., the F-dual predictable projection or compensator of $\mu$ ). The basis B is supposed to satisfy «the usual conditions».

Moreover here we denote $\mathscr{I}(b, \nu)$ the class of 1-stable Lévy motions with Lévy measure $\nu(d x)=1_{E}(x) c x^{-2} d x$ and drift $b \cdot t$, where $b, c$ are real numbers, $c \geqslant 0$ and $E=\mathbf{R}-\{0\}$.

Without any further statement, all the notions (predictability, stochastic integrals etc.) and the processes to be considered in the present section, will be implicitly referred to the fixed basis $\mathbf{B}$.

Proposition. Let $(X, \mathbf{F})$ be a semimartingale. Assume that there exist a predictable process $H$ and a process $(Z, \mathbf{F}), Z \in \mathscr{I}(b, \nu)$, such that

$$
X_{t}=\int_{[0, t]}\left|H_{s}\right| d Z_{s} \quad\left(\text { resp., } X_{t}=\int_{[0, t]} H_{s} d Z_{s}\right), \quad t \geqslant 0 .
$$


Then the following decomposition holds

$$
X_{t}=\int_{[0, t]} \int_{|x| \leqslant 1} x(\mu-\pi)(d s, d x)+\int_{\mid 0, t]} \int_{|x|>1} x \mu(d s, d x)+b A_{t}, \quad t \geqslant 0,
$$

with $\pi(d s, d x)=\left|H_{s}(\omega)\right| d s \otimes \nu(d x)$ and $A_{t}(\omega)=\int_{0}^{t}\left|H_{s}(\omega)\right| d s$ (resp., $A_{t}(\omega)=$ $\left.\int_{0}^{t} H_{s}(\omega) d s\right)$.

$\mathrm{P}$ r o o f. If $\nu=0, Z$ is P-equal to a pure drift, i.e., $Z_{t}=b \cdot t$ for all $t$ and the conclusion is trivial.

So assume that $c>0$ and that the left-hand side representation formula holds. Denote by $p$ the jump-measure of $Z: p$ is Poisson with compensator $q(d s, d x)=d s \otimes \nu(d x)$ and for all $t$

$$
Z_{t}=\int_{[0, t]} \int_{|x| \leqslant 1} x(p-q)(d s, d x)+\int_{] 0, t]} \int_{|x|>1} x p(d s, d x)+b \cdot t .
$$

The assumption implies that the jump-measures $\mu, p$ of $X, Z$, respectively, are linked as follows:

$$
\mu(\omega ; A)=\int 1_{A}\left(t,\left|H_{t}(\omega)\right| \cdot y\right) p(\omega ; d t, d y), \quad A \in \mathscr{B}\left(\mathbf{R}_{+}\right) \otimes \mathscr{E}
$$

and by the change of variable $x=\left|H_{s}(\omega)\right| \cdot y$ it is easy to check that $\pi$ can be factorized as follows:

$$
\pi(\omega ; d s, d x)=|H(s, \omega)| q(d s, d x) .
$$

On the other hand the continuous martingale part of $X$ is $\mathbf{P}$-equal to the null process (cf. [3, Chapter II, $2, \S \mathrm{f}]$ ) and $X$ can be represented in the following canonical form (see [1], [4]):

$$
X_{t}=\int_{\mid 0, t]} \int_{|x| \leqslant 1} x(\mu-\pi)(d s, d x)+\int_{j 0, t]} \int_{|x|>1} x \mu(d s, d x)+B_{t}, \quad t \geqslant 0,
$$

where $B$ is a process having locally finite variation paths which are continuous, thanks to (1.3) ( $B$ is the first local characteristic of $X$ associated to the function $h(x)=x 1_{\{|x| \leqslant 1\}}$; cf. [3, Chapter III, $2, \S \mathrm{c}])$.

In order to conclude we have to prove that

$$
B_{t}=b A_{t} \quad \text { for all } t
$$

up to P-equality.

Similarly to (3.7) in [7] set now

$$
\begin{aligned}
N_{t} & =\int_{j 0, t]} \int_{|x| \leqslant 1} x(p-q)(d s, d x), & K_{t} & =\int_{\mid 0, t]} \int_{|x|>1} x p(d s, d x), \\
C_{t} & =\int_{[0, t]}\left|H_{s}\right| d N_{s}, & W_{t} & =\int_{\mid 0, t]}\left|H_{s}\right| d K_{s},
\end{aligned}
$$

so that, for all $t, Z_{t}=N_{t}+K_{t}+b t$, and thanks to the assumption

$$
X_{t}=C_{t}+W_{t}+b A_{t} .
$$

But relation (1.4) can be rewritten as follows:

$$
X_{t}=M_{t}+V_{t}+B_{t}, \quad t \geqslant 0,
$$

where

$$
M_{t}=\int_{j 0, t]} \int_{|x| \leqslant 1} x(\mu-\pi)(d s, d x), \quad V_{t}=\int_{j 0, t]} \int_{|x|>1} x \mu(d s, d x),
$$

so that (1.5) is equivalent to the relation $C_{t}+W_{t}=M_{t}+V_{t}$, i.e.,

$$
M_{t}+\left(V_{t}-W_{t}\right)-C_{t}=0 \quad \text { for all } t,
$$

up to $\mathbf{P}$-equality.

Lemma 1.1. The process $V-W$ is a purely discontinuous local martingale. 
P r o o f. It suffices to proceed exactly as for the proof of Lemma 2 in [7]: in fact that proof is based there on the relation (3.3) which is to be replaced here by the similar relation (1.2). (In [7] $p$ depends on $\left(\omega, \omega^{\prime}\right)$ but such dependence is irrelevant to the proof.)

It follows that $M+(V-W)-C$ is a purely discontinuous local martingale. Because of (1.4), $X$ has all its jumps in $M+V$; and because of (1.7) $X$ has all its jumps in $C+W$ too. Thus, up to P-equality

$$
\Delta X=\Delta(M+V)=\Delta(C+W) .
$$

As a consequence, $M+(V-W)-C=(M+V)-(C+W)$ is a purely discontinuous local martingale having jump-process $\mathbf{P}$-equal to zero. We conclude that $M+(V-W)-C$ is $\mathbf{P}$-equal to zero and we are done.

When the right-hand side representation formula holds still with $c>0$, the proof is performed exactly as above starting from the relations

$$
\begin{gathered}
\mu(\omega ; A)=\int 1_{A}\left(t, H_{t}(\omega) \cdot y\right) p(\omega ; d t, d y), \quad A \in \mathscr{B}\left(\mathbf{R}_{+}\right) \otimes \mathscr{E}, \\
\pi(\omega ; d s, d x)=H(s, \omega) q(d s, d x),
\end{gathered}
$$

the latter being easily derived from the former one by the change of variable $x=H_{s}(\omega) \cdot y$. The proposition is proved.

By combining the above proposition and Theorem 1 in [7] we obtain the following theorem which completes the characterization of the real-valued semimartingales which can be represented as a stochastic integral with respect to a strictly 1-stable Lévy process.

Theorem. a) Let $X$ be a $\mathbf{F}$-semimartingale. Assume that there exist a predictable process $H$ and a measure $\nu(d x)=1_{E}(x) c x^{-2} d x, c \geqslant 0$, such that

$$
\pi(\omega ; d s, d x)=\left|H_{s}(\omega)\right| d s \otimes \nu(d x)
$$

and for all $t$

$$
X_{t}=\int_{[0, t]} \int_{|x| \leqslant 1} x(\mu-\pi)(d s, d x)+\int_{j 0, t]} \int_{|x|>1} x \mu(d s, d x)+b A_{t},
$$

where $b$ is a real number and $A_{t}(\omega)=\int_{0}^{t}\left|H_{s}(\omega)\right| d s$ (respectively, $\left.A_{t}(\omega)=\int_{0}^{t} H_{s}(\omega) d s\right)$.

Then there exists $Z \in \mathscr{I}(b, \nu)$, possibly defined on an extension of the original probability space, such that

$$
X_{t}=\int_{j 0, t]}\left|H_{s}\right| d Z_{s} \quad\left(\text { resp., } \quad X_{t}=\int_{j 0, t]} H_{s} d Z_{s}\right) .
$$

b) Let $(X, \mathbf{F})$ be a semimartingale such that there exist a predictable process $H$ and $(Z, \mathbf{F}), Z \in \mathscr{I}(b, \nu)$, permitting the representation formula

$$
X_{t}=\int_{j 0, t]}\left|H_{s}\right| d Z_{s} \quad\left(\text { resp., } \quad X_{t}=\int_{j 0, t]} H_{s} d Z_{s}\right), \quad t \geqslant 0 .
$$

Then $\pi(d s, d x)=\left|H_{s}(\omega)\right| d s \otimes \nu(d x)$ and $X$ can be decomposed as in a) with $A_{t}(\omega)=$ $\int_{0}^{t}\left|H_{s}(\omega)\right| d s$ (resp., $\left.A_{t}(\omega)=\int_{0}^{t} H_{s}(\omega) d s\right)$.

2. Elementary construction of the extension. With reference to the proof of Theorems 1 and 2 in [7], here we want to point out a more elementary way of proceeding, not using more than the concept of dual predictable projection of a random measure.

Recall Assumptions 1 and 2 in [7].

Assumption 1. There exist a predictable process $H(s, \omega)$ and constants $c_{+} \geqslant 0$, $c_{-} \geqslant 0,0<\alpha<2$, such that

$$
\pi(\omega, d s, d x)=|H(s, \omega)|^{\alpha} q(d s, d x),
$$

where $q(d s, d x)=d s \otimes \nu(d x), \nu(d x)=1_{\mid 0,+\infty[}(x) c_{+} x^{-(1+\alpha)} d x+1_{-\infty, 0[}(x) c_{-}|x|^{-(1+\alpha)} d x$.

Assumption 2. The same as in the preceding assumption but with a symmetric $\nu$ (i.e., $c_{+}=c_{-}=c \geqslant 0$ ).

First we assume that $X$ verifies Assumption 1 with $c_{+}+c_{-}>0$ (recall that $c_{+}=c_{-}$ when $\alpha=1$ ). 
With reference to statements a) of Theorem 1 and a), b) of Theorem 2 in [7], a crucial point for the proof is the construction of a stochastic basis $\overline{\mathbf{B}}$ verifying (3.2) and (3.3) in [7], i.e., an extension of $\mathbf{B}, \overline{\mathbf{B}}=(\bar{\Omega}, \overline{\mathscr{F}}, \overline{\mathbf{F}}, \overline{\mathbf{P}})$ defined by means of an auxiliary space $\Omega^{\prime}$ with

$$
\left\{\begin{array}{l}
\bar{\Omega}=\Omega \times \Omega^{\prime}, \quad \mathbf{P}=\varphi(\overline{\mathbf{P}}), \\
\varphi: \bar{\Omega} \rightarrow \Omega, \quad \varphi\left(\omega, \omega^{\prime}\right)=\omega \quad \text { for all }\left(\omega, \omega^{\prime}\right),
\end{array}\right.
$$

extension which carries a Poisson random measure $p\left(\left(\omega, \omega^{\prime}\right) ; \cdot\right)$ on $\mathscr{B}\left(\mathbf{R}_{+}\right) \otimes \mathscr{E}$ with the compensator $q(d t, d y)$, such that one has, for $\overline{\mathbf{P}}$-a.e. $\left(\omega, \omega^{\prime}\right)$,

$$
\mu(\omega ; A)=\int 1_{A}\left(t,\left|H_{t}(\omega)\right| y\right) p\left(\left(\omega, \omega^{\prime}\right) ; d t, d y\right) \quad \text { if } \quad A \in \mathscr{B}\left(\mathbf{R}_{+}\right) \otimes \mathscr{E} .
$$

Such construction can be performed as follows. Consider any stochastic basis $\mathbf{B}^{\prime}=\left(\Omega^{\prime}, \mathscr{F}^{\prime}, \mathbf{F}^{\prime}, \mathbf{P}^{\prime}\right), \mathbf{F}^{\prime}=\left(\mathscr{F}_{t}^{\prime}\right)_{t \geqslant 0}$ which carries a strictly $\alpha$-stable Lévy process $\left(Z^{\prime}, \mathbf{F}^{\prime}\right)$ with Lévy measure $\nu(d x)$ and jump-measure $p^{\prime}\left(\omega^{\prime} ; d s, d x\right)$ : define a standard extension

$$
\overline{\mathbf{B}}=(\bar{\Omega}, \bar{F}, \overline{\mathbf{F}}, \overline{\mathbf{P}})
$$

by setting $\bar{\Omega}=\Omega \times \Omega^{\prime}, \overline{\mathbf{P}}=\mathbf{P} \otimes \mathbf{P}^{\prime}, \overline{\mathscr{F}}$ being the $\overline{\mathbf{P}}$-completion of $\mathscr{F} \otimes \mathscr{F}^{\prime}$ and $\overline{\mathbf{F}}=\left(\overline{\mathscr{F}}_{t}\right)_{t \geqslant 0}$ the usual $\overline{\mathbf{P}}$-augmentation of the filtration $\left(\check{\mathscr{F}}_{t}\right)_{t \geqslant 0}$, where $\check{\mathscr{F}}_{t}=\cap_{s>t} \mathscr{F}_{s} \otimes \mathscr{F}_{s}^{\prime}$.

We also consider the functions $\varphi: \bar{\Omega} \rightarrow \Omega, \psi: \bar{\Omega} \rightarrow \Omega^{\prime}$ defined respectively by $\varphi\left(\omega, \omega^{\prime}\right)=\omega, \psi\left(\omega, \omega^{\prime}\right)=\omega^{\prime}$, so that the given processes and measures are naturally extended to $\bar{\Omega}$ by considering $X \circ \varphi, \mu \circ \varphi, H \circ \varphi, p^{\prime} \circ \psi$ etc.

Now add the point $\delta=\infty$ to $E$ and denote by $E_{\delta}$ the locally compact space $E \cup\{\delta\}$, by $\mathscr{E}_{\delta}$ the Borel $\sigma$-algebra of $E_{\delta}$.

Adopting the convention $x /\left|H_{s}(\omega)\right|=\delta$ whenever $x \in E$ and $H_{s}(\omega)=0$, define a $\left(\mathbf{R}_{+} \times E_{\delta}\right)$-valued random measure $\bar{p}$ on $\bar{\Omega}$ as follows:

$$
\left\{\begin{array}{l}
\bar{p}\left(\left(\omega, \omega^{\prime}\right) ; d s, d x\right)=p_{1}\left(\left(\omega, \omega^{\prime}\right) ; d s, d x\right)+p_{2}(\omega ; d s, d x), \text { where } \\
p_{1}\left(\left(\omega, \omega^{\prime}\right) ; d s, d x\right)=1_{\left\{H_{s}(\omega)=0\right\}} p^{\prime}\left(\omega^{\prime} ; d s, d x\right), \\
p_{2}(\omega ;[0, t] \times B)=\int_{[0, t] \times E_{\delta}} 1_{B}\left(\frac{x}{\left|H_{s}(\omega)\right|}\right) 1_{\left\{H_{s}(\omega) \neq 0\right\}} \mu(\omega ; d s, d x), \\
B \text { denoting any set in } \mathscr{E}_{\delta} .
\end{array}\right.
$$

Note that $p_{1}$ is concentrated on $\mathbf{R}_{+} \times E$ and that, for all $\omega, t$ we have $p_{2}(\omega ;[0, t] \times\{\delta\})=0$ so that $p_{2}\left(\omega ; \mathbf{R}_{+} \times\{\delta\}\right)=0$. It follows that for all $\left(\omega, \omega^{\prime}\right)$ the measure $\bar{p}$ charges only $\mathbf{R}_{+} \times E$ and we may identify $\bar{p}$ with its restriction $p$ to $\left(\mathbf{R}_{+} \times E, \mathscr{B}\left(\mathbf{R}_{+}\right) \otimes \mathscr{E}\right)$.

Lemma 2.2. The measure $p$ is Poisson on $\overline{\mathbf{B}}$ with $q(d s, d x)$ as a $\overline{\mathbf{B}}$-compensator.

P r o o f. Let $\bar{P}$ denote the $\overline{\mathbf{F}}$-predictable $\sigma$-algebra in $\mathbf{R}_{+} \times \bar{\Omega}$ and remark that $p^{\prime} \circ \psi$ is a $\overline{\mathscr{P}} \otimes \mathscr{E}-\sigma$-finite integer-valued random measure in the sense of [5] (cf. [5, Chapter II, 1]) owing to Proposition (10.47), (a) in [3], so that the same holds for $p_{1}$ since $H \circ \varphi$ is $\overline{\mathscr{P}}$-measurable and clearly, for all $\left(\omega, \omega^{\prime}\right)$

$$
p_{1}\left(\left(\omega, \omega^{\prime}\right) ; \cdot\right)=1_{\left\{(H \circ \varphi)_{s}\left(\omega, \omega^{\prime}\right)=0\right\}} p^{\prime} \circ \psi\left(\left(\omega, \omega^{\prime}\right) ; \cdot\right) .
$$

Hence $p_{1}$ admits a $\overline{\mathbf{B}}$-compensator $q_{1}$ due to Theorem 1.8, p. 120 in $[5$, v. 1] and it is easy to check that $q_{1}(d s, d x)=1_{\left\{H_{s}(\omega)=0\right\}} q(d s, d x)$. Indeed, thanks to Proposition (10.47), (b) in [3], $q \circ \psi=q$ is the $\overline{\mathbf{B}}$-compensator of $p^{\prime} \circ \psi$ so that, taking the above relation into account, the measure $1_{\left\{(H \circ \varphi)_{s}\left(\omega, \omega^{\prime}\right)=0\right\}} q(d s, d x)=1_{\left\{H_{s}(\omega)=0\right\}} q(d s, d x)$ turns out to be the $\overline{\mathbf{B}}$-compensator of $p_{1}$.

Because of the definition of $p$, in order to conclude, clearly it suffices to verify that the $p_{2}$-restriction to $\mathbf{R}_{+} \times E$ admits

$$
q_{2}(\omega ; d s, d x)=1_{\left\{H_{s}(\omega) \neq 0\right\}} q(d s, d x)
$$

as a $\overline{\mathbf{B}}$-compensator. 
In fact, fix any compact set $K$ in $\mathscr{E}$ and define $N_{t}(\omega)=p_{2}([0, t] \times K)-q_{2}([0, t] \times K)$. Whenever $F \in \mathscr{F}_{u}$ and $u<t$, from Assumption 1 it follows

$$
\begin{aligned}
& \left.\left.\mathbf{E}_{\mathbf{P}}\left[1_{F} p_{2}(] u, t\right] \times K\right)\right] \\
& =\int_{\Omega} d \mathbf{P}(\omega)\left(\int_{\mid u, t] \times E} 1_{F}(\omega) 1_{\left\{H_{s}(\omega) \neq 0\right\}} 1_{K}\left(\frac{x}{\left|H_{s}(\omega)\right|}\right) \mu(\omega ; d s, d x)\right) \\
& =\int_{\Omega} d \mathbf{P}(\omega) 1_{F}(\omega)\left(\int_{\mid u, t]}\left|H_{s}(\omega)\right|^{\alpha} 1_{\left\{H_{s}(\omega) \neq 0\right\}} d s\left(\int_{E} 1_{K}\left(\frac{x}{\left|H_{s}(\omega)\right|}\right) \nu(d x)\right)\right),
\end{aligned}
$$

so that, by the change of variable $y=x /\left|H_{s}(\omega)\right|$ we have

$$
\begin{aligned}
\left.\left.\mathbf{E}_{\mathbf{P}}\left[1_{F} p_{2}(] u, t\right] \times K\right)\right] & =\int_{\Omega} d \mathbf{P}(\omega) 1_{F}(\omega)\left(\int_{] u, t]} 1_{\left\{H_{s}(\omega) \neq 0\right\}} d s\left(\int_{E} 1_{K}(y) \nu(d y)\right)\right) \\
& \left.\left.=\mathbf{E}_{\mathbf{P}}\left[1_{F} q_{2}(] u, t\right] \times K\right)\right]<\infty
\end{aligned}
$$

hence, $N$ is a $\mathbf{B}$-martingale.

By a standard proof (cf. [2, Lemma 3.1, p. 67]) it follows that $\int_{[0, \cdot]} f_{s} d N_{s}$ is a $\mathbf{B}-$ martingale for all bounded $\mathscr{P}$-measurable functions $f, \mathscr{P}$ denoting the F-predictable $\sigma$-algebra in $\mathbf{R}_{+} \times \Omega$. A monotone class argument permits to conclude that

$\int_{\Omega} d \mathbf{P}(\omega)\left(\int_{\mathbf{R}_{+} \times E} f(s, \omega, x) p_{2}(\omega ; d s, d x)\right)=\int_{\Omega} d \mathbf{P}(\omega)\left(\int_{\mathbf{R}_{+} \times E} f(s, \omega, x) q_{2}(\omega ; d s, d x)\right)$

for every $\mathscr{P} \otimes \mathscr{E}$-measurable and positive function $f$ on $\mathbf{R}_{+} \times E$.

This property expresses the fact that the measure $q_{2}$ is the B-compensator of the $p_{2}$-restriction to $\mathbf{R}_{+} \times E$. Still because of Proposition (10.47) in [3], the $\left(p_{2} \circ \varphi\right)$-restriction to $\mathbf{R}_{+} \times E$ admits $q_{2} \circ \varphi=1_{\left\{(H \circ \varphi)_{s}\left(\omega, \omega^{\prime}\right)=0\right\}} q(d s, d x)=1_{\left\{H_{s}(\omega)=0\right\}} q(d s, d x)$ as a $\overline{\mathbf{B}}$-compensator. Lemma 2 is proved.

It remains only to check that $\mu$ and $p$ are linked by formula (2.1).

In fact, recalling the definition of $p$, for all $A$ in $\mathscr{B}\left(\mathbf{R}_{+}\right) \otimes \mathscr{E}$ and all $\left(\omega, \omega^{\prime}\right)$

$$
\begin{aligned}
\int 1_{A}\left(t,\left|H_{t}(\omega)\right| y\right) p\left(\left(\omega, \omega^{\prime}\right) ; d t, d y\right) & =\int 1_{A}\left(t,\left|H_{t}(\omega)\right| y\right) 1_{\left\{H_{t}(\omega) \neq 0\right\}} p\left(\left(\omega, \omega^{\prime}\right) ; d t, d y\right) \\
& =\int_{\mathbf{R}_{+} \times E} 1_{A}\left(t,\left|H_{t}(\omega)\right| y\right) 1_{\left\{H_{t}(\omega) \neq 0\right\}} p_{2}(\omega ; d t, d y) \\
& =\int_{\mathbf{R}_{+} \times E} 1_{A}(t, x) 1_{\left\{H_{t}(\omega) \neq 0\right\}} \mu(\omega ; d t, d x) .
\end{aligned}
$$

But Assumption 1 permits to see that one has, for P-almost every $\omega$

$$
\int_{\mathbf{R}_{+} \times E} 1_{A}(t, x) 1_{\left\{H_{t}(\omega) \neq 0\right\}} \mu(\omega ; d t, d x)=\int_{\mathbf{R}_{+} \times E} 1_{A}(t, x) \mu(\omega ; d t, d x)
$$

independently of $A$ in $\mathscr{B}\left(\mathbf{R}_{+}\right) \otimes \mathscr{E}$. Relation (2.1) follows. Then the proof can be completed exactly as in [7].

When statement b) (resp., c)) of Theorem 1 (resp., 2) is concerned, Assumption 2 is in force and in a similar way one constructs an extension $\mathbf{B}^{*}=\left(\Omega^{*}, \mathscr{F}^{*}, \mathbf{F}^{*}, \mathbf{P}^{*}\right)$ which carries a Poisson random measure $p^{*}$ with the compensator $q(d t, d y)$ such that formula (3.5) in [7] holds.

Indeed let $\mathbf{B}^{\prime}=\left(\Omega^{\prime}, \mathscr{F}^{\prime}, \mathbf{F}^{\prime}, \mathbf{P}^{\prime}\right)$ denote a basis carrying a strictly $\alpha$-stable Lévy process $\left(Z^{\prime}, \mathbf{F}^{\prime}\right)$ with (symmetric) Lévy measure $\nu(d x)$ and jump-measure $p^{\prime}\left(\omega^{\prime} ; d s, d x\right)$ : from such a basis we build up a standard extension $\mathbf{B}^{*}$ of $\mathbf{B}$ exactly as in the above case.

Then, unlike (2.2), we set

$$
\begin{aligned}
p\left(\left(\omega, \omega^{\prime}\right) ;[0, t] \times B\right)= & \int_{j 0, t\} \times E_{\delta}} 1_{B}(x) 1_{\left\{H_{s}(\omega)=0\right\}} p^{\prime}\left(\omega^{\prime} ; d s, d x\right) \\
& +\int_{[0, t] \times E_{\delta}} 1_{B}\left(\frac{x}{H_{s}(\omega)}\right) 1_{\left\{H_{s}(\omega) \neq 0\right\}} \mu(\omega ; d s, d x),
\end{aligned}
$$

for all $t$ and $B$ in $\mathscr{E}_{\delta},\left(E_{\delta}, \mathscr{E}_{\delta}\right)$ being defined as above. 
Denote by $p^{*}$ the restriction of $p$ to $\left(\mathbf{R}_{+} \times E, \mathscr{B}\left(\mathbf{R}_{+}\right) \otimes \mathscr{E}\right)$ : measure $p^{*}$ is shown to verify the analog of Lemma 2 and, thanks to the latter definition, it is easy to verify that, for $\mathbf{P}^{*}$-a.e. $\left(\omega, \omega^{\prime}\right)$,

$$
\mu(\omega ; A)=\int 1_{A}\left(t, H_{t}(\omega) y\right) p^{*}\left(\left(\omega, \omega^{\prime}\right) ; d t, d y\right) \quad \text { if } \quad A \in \mathscr{B}\left(\mathbf{R}_{+}\right) \otimes \mathscr{E}
$$

which is formula (3.5) in [7].

Also in this case the proof is completed exactly as in [7].

\section{REFERENCES}

1. Григелионис Б. О мартингальной характеризации случайных процессов с независимыми приращениями. - Литов. матем. сб., 1977, т. 17, № 1, с. 53-60.

2. Ватанабэ $C$., Икэда $H$. Стохастические дифференциальные уравнения и диффузионные процессы. М.: Наука, 1986, 445 с.

3. Jacod J. Calcul Stochastique et Problèmes de Martingales. Berlin: Springer-Verlag, 1979,539 p. (Lecture Notes in Math., v. 714.)

4. Jacod J., Mémin J. Caractéristiques locales et conditions de continuité absolue pour les semimartingales. - Z. Wahrscheinlichkeitstheor. verw. Geb., 1976, v. 35, p. 1-37.

5. Жакод Ж., Ширяев А. Н. Предельные теоремы для случайных процессов. Т. 1, 2. М.: Физматлит, 1994,544 c; 368 с.

6. Кабанов Ю.М., Липчер Р.Ш., Ширяев А.Н. О представлении целочисленных случайных мер и локальных мартингалов с помошью случайных мер с детерминированными компенсаторами. - Матем. сб., 1980, т. 111, № 2, с. 293-307.

7. Zanzotto $P$. A. Representation of a class of semimartingales as stable integrals. Теория вероятн. и ее примен., 1998, т. 43, в. 4, с. 808-818.

8. Zanzotto P. A. On stochastic differential equations driven by Cauchy process and the other stable Lévy motions. - Ann. Probab., 2002, v. 30, № 2, p. 802-825.

Поступила в редакцию 17.V. 2000 